\title{
Sociologia da Odontologia: aspectos da densidade da relação habitante/cirurgião- dentista/PIB per capita em municípios do interior paulista com cursos de graduação em Odontologia
}

\author{
Sociology of Dentistry: aspects of dentist-population-GDP ratio in cities of the interior of São Paulo state with \\ undergraduate courses in Dentistry \\ Sociología de la odontología: aspectos de la relación dentista-población-PIB en ciudades del interior del estado \\ de São Paulo con cursos de graduación en Odontología \\ Maria Cristina Rosifini ALVES REZENDE ${ }^{1}$ \\ Moacir José ROSSINI NETO ${ }^{1}$ \\ João Pedro Justino de Oliveira LIMÍRIO² \\ ${ }^{I}$ Faculdade de Odontologia de Araçatuba, Universidade Estadual Paulista (UNESP) 16015-050 Araçuba-SP, Brasil \\ ${ }^{2}$ Programa de Pós-Graduação em Odontologia, Faculdade de Odontologia de Araçatuba, Universidade Estadual Paulista (UNESP) 16015-050 Araçatuba-SP, Brasil
}

\begin{abstract}
Resumo
Introdução: Na Odontologia, novos modelos profissionais tais como empresariamento, proletarização e maior concorrência redefinem não só a relação profissional/paciente outrora individualizada, como também a direção do fluxo migratório do cirurgião-dentista no espaço geográfico. Cidades que abrigam um campus universitário transformam o seu entorno imediato social, cultural e econômico, na medida em que dinamizam o surgimento de novas atividades e investimentos. Além disso, o conhecimento produzido dentro dos muros acadêmicos coloca a instituição de ensino como principal agente no desenvolvimento e transformação do município, atrativo polo formador científico e tecnológico. Com base no exposto, o propósito deste trabalho foi relacionar a densidade da relação de habitantes/cirurgião-dentista/PIB per capita em municípios do interior paulista com cursos de graduação em Odontologia. Material e Método: O levantamento de dados relativos à densidade de cirurgiões-dentistas por população foi realizado com base nos dados estatísticos do CROSP- Conselho Regional de Odontologia do Estado de São Paulo; a quantidade e a distribuição dos cursos de Odontologia no interior do estado de São Paulo foram coletadas nos dados do MEC - Ministério da Educação e Cultura e o PIB per capita foi coletado nos dados estatísticos do Portal de Estatísticas do Estado de São Paulo SEADE. Resultados: No interior do estado de São Paulo os Cursos de Odontologia estão distribuídos em 32 municípios, oferecidos por 48 instituições, somando 5800 vagas. Esses municípios concentram 26.397 (30\%) dos registros profissionais paulistas. A densidade número de habitantes/cirurgião-dentista foi maior em Itapeva (816) e menor em Santos (205). Os maiores valores de PIB per capita não corresponderam ao maior número de cirurgiões-dentistas e municípios com valores próximos em reais, tais como Ribeirão Preto (R $\$ 45,789)$ e Itu (R $\$ 46,661)$ diferiram amplamente no registro, com 2247 cirurgiõesdentistas registrados em Ribeirão Preto contra 266 profissionais em Itu. Conclusão: Os Cursos de Odontologia instalados no interior paulista embora dinamizem a economia dos 32 municípios onde estão instalados, não respondem isoladamente pela concentração de cirurgiões-dentistas nesses espaços geográficos. Outros fatores tais como capital humano, qualidade de vida, instituições, ambiente de negócios, mercados e recursos naturais também devem ser considerados.
\end{abstract}

Descritores: Faculdades de Odontologia; Mercado de Trabalho; Desenvolvimento Econômico.

\section{Abstract}

Introduction: In dentistry, new professional models such as entrepreneurship, proletarianization and greater competition redefine not only the previously individualized professional / patient relationship, but also the direction of migratory flow of the dental surgeon in the geographic space. Cities that house a university campus transform their immediate social, cultural and economic environment, as they stimulate the emergence of new activities and investments. In addition, the knowledge produced within the academic walls places the educational institution as the main agent in the development and transformation of the municipality, attractive polo scientific and technological trainer. In the interior of the state of São Paulo, the Dentistry Courses are distributed in 32 municipalities, offered by 48 institutions, totaling 5800 vacancies. Based on the above, the purpose of this study was to correlate the density of inhabitants / dentist / per capita GDP ratio in municipalities in the interior of São Paulo with undergraduate courses in dentistry. Material and Methods: Data collection on the density of dental surgeons by population was carried out based on the statistical data of CROSP - Regional Council of Dentistry of the State of São Paulo; the quantity and distribution of Dentistry courses in the interior of the State of São Paulo were collected from the MEC - Ministry of Education and Culture data and the per capita GDP was collected from the Statistical Portal of the State of São Paulo - SEADE. Results: Dentist/population ratio was higher in Itapeva (816) and lower in Santos (205). Per capita GDP and the number of dentists did not show a positive correlation. In cities with close values in reais, such as Ribeirão Preto ( $\mathrm{R} \$ 45,789$ ) and Itu ( $\$$ \$ 46,661), registered professionals differ widely, with 2247 dentists registered in Ribeirão Preto versus 266 professionals in Itu. Conclusion: Dentistry courses installed in the interior of São Paulo, although can stimulate of the economy of the 32 municipalities where they are installed do not respond in isolation to the concentration of dental surgeons in these geographical spaces. Other factors such as human capital, quality of life, institutions, business environment, markets and natural resources should be considered.

Descriptors: Schools, Dental; Job Market; Economic Development.

\section{Resumen}

En la Odontología, nuevos modelos profesionales tales como empresariado, proletarización y mayor competencia redefinen no sólo la relación profesional / paciente otrora individualizada, sino también la dirección del flujo migratorio del cirujano-dentista en el espacio geográfico. Las ciudades que albergan un campus universitario transforman su entorno inmediato social, cultural y económico, en la medida en que dinamizan el surgimiento de nuevas actividades e inversiones. Además, el conocimiento producido dentro de los muros académicos coloca a la institución de enseñanza como principal agente en el desarrollo y transformación del municipio, atractivo por el formador científico y tecnológico. En el interior del estado de São Paulo los Cursos de Odontología están distribuidos en 32 municipios, ofrecidos por 48 instituciones, sumando 5800 vacantes. En base a lo expuesto, el propósito de este trabajo fue relacionar la densidad de la relación de habitantes / cirujano-dentista / PIB per cápita en municipios del interior paulista con cursos de graduación en Odontología. Material y Método: El levantamiento de datos relativos a la densidad de cirujanos-dentistas por población fue realizado con base en los datos estadísticos del CROSPConsejo Regional de Odontología del Estado de São Paulo; la cantidad y la distribución de los cursos de Odontología en el interior del estado de São Paulo fueron recolectados en los datos del MEC - Ministerio de Educación y Cultura y el PIB per cápita fue recolectado en los datos estadísticos del Portal de Estadísticas del Estado de São Paulo - SEADE. Resultados: No interior del estado de São Paulo o Cursos de Odontología están distribuidos en 32 municipios, ofrecidos por 48 instituciones, 5800 vagas. Eses municipios concentran 26.397 (30\%) dos registros profesionales paulistas. El número de habitantes/odontólogos fue mayor en Itapeva (816) y menor en Santos (205). Otros valores de PIB per cápita no corresponderán a un número mayor de odontólogos y municípios con valores similares como Ribeirão Preto (R \$ 45.789) e Itu (R \$ 46.661), mostraran diferencia de profesionales registrados con 2247 en Ribeirão Preto contra 266 en Itu. Conclusión: Os Cursos de Odontología aunque promueven el desarrollo de la economía en 32 municipios donde están instalados, no responda aisladamente por la concentración de profesionales. Otros factores como el capital humano, calidad de vida, instituciones, entorno empresarial, mercados y recursos naturales deben considerarse.

Descriptores: Faculdade de Odontología; Mercado de Trabajo; Desarrollo Económico.

\section{INTRODUÇÃO}

Na Odontologia, novos modelos profissionais tais como empresariamento, proletarização e maior concorrência redefinem não só a relação profissional/paciente, outrora individualizada, como também a direção do fluxo migratório do cirurgião-dentista no espaço geográfico, na busca de inserção nos mercados com demandas latentes por assistência odontológica da sociedade atual ${ }^{1}$.

De um modo geral, a cidades que abrigam um campus universitário transformam o seu entorno 
imediato social, cultural e econômico. Segundo Schneider ${ }^{2}$ a universidade é um especial atrativo para o surgimento de novas atividades e investimentos nos municípios onde é instalada. Isso decorre do volume considerável de recursos injetados, seja por meio do salário do quadro de recursos humanos da instituição, seja por meio do consumo efetuado pelos acadêmicos, o que desencadeia um efeito multiplicador para a economia do município. Além disso, o conhecimento produzido dentro dos muros acadêmicos, utilizado e aplicado pela comunidade, coloca a instituição de ensino como principal agente na busca pela inovação, desenvolvimento e transformação regional ${ }^{3}$, transformando essas regiões em polos formadores científicos e tecnológicos.

Os Cursos de Odontologia do interior do estado de São Paulo figuram entre os mais importantes do país. Distribuídos em 32 municípios, dinamizam as economias locais ${ }^{4}$ gerando emprego e renda, delineando novos matizes nas relações de trabalho. Além disso, Cardoso et al. ${ }^{5}$ apontam que os polos formadores atraem a população de maior poder aquisitivo, justificando a permanência de muitos cirurgiões-dentistas nesses locais.

Com base no exposto, o propósito deste trabalho é relacionar a densidade da relação de habitantes/cirurgião-dentista/PIB per capita em municípios do interior paulista com cursos de graduação em Odontologia ao mercado de trabalho odontológico.

\section{MATERIAL E MÉTODO}

Foi realizado estudo transversal descritivo com utilização de dados secundários e de domínio público do Conselho Regional de Odontologia de São Paulo (CROSP) ${ }^{6}$ e Ministério da Educação e Cultura (MEC). A coleta de dados foi realizada de abril a outubro de 2018.

O levantamento de dados relativos à densidade de cirurgiões-dentistas por população foi realizado com base nos dados estatísticos do Conselho Regional de Odontologia do estado de São Paulo $(\mathrm{CROSP})^{6}$, consultando o número de profissionais cadastrados em cada cidade e o número de habitantes por cirurgião-dentista. Além disso, a quantidade e a distribuição dos cursos de Odontologia no interior do estado de São Paulo foram coletadas nos dados do Ministério da Educação e Cultura (MEC) ${ }^{7}$. O PIB per capita foi coletado nos dados estatísticos do Portal de Estatísticas do estado de São Paulo (SEADE) ${ }^{8}$. Os dados foram tabulados usando o software Microsoft Excel 2013 (EUA, Microsoft (C), onde foram analisados de forma descritiva.

\section{RESULTADOS E DISCUSSÃO}

A Tabela 1 apresenta os resultados dos Cursos de Odontologia no interior do estado de São Paulo. Foram identificados 48 cursos em 32 dos 645 municípios paulistas, sendo possível observar que são majoritariamente instituições privadas $(85.5 \%)$. Com relação à organização acadêmica, $60.41 \%$ são Universidades.

Tabela 1: Distribuição dos Cursos de Odontologia no interior paulista por município, instituição, categoria administrativa e organização acadêmica

\begin{tabular}{|c|c|c|c|}
\hline Município & Instituição & $\begin{array}{l}\text { Categoria } \\
\text { Administrativa }\end{array}$ & $\begin{array}{l}\text { Organização } \\
\text { Acadêmica }\end{array}$ \\
\hline Adamantina & FAI & Pública Municipal & Centro Universitário \\
\hline Araçatuba & UNESP & Pública Estadual & Universidade \\
\hline \multirow[b]{2}{*}{ Araraquara } & UNESP & Pública Estadual & Universidade \\
\hline & UNIARA & Privada $\mathrm{SFL}^{*}$ & Universidade \\
\hline Araras & \begin{tabular}{|l} 
UNIARARAS \\
FHO
\end{tabular} & Privada $\mathrm{SFL}^{*}$ & Centro Universitário \\
\hline Avaré & UNIFSP & Privada CFL ${ }^{* *}$ & Centro Universitário \\
\hline Barretos & UNIFEB & Privada SFL* & Centro Universitário \\
\hline \multirow[b]{2}{*}{ Bauru } & USP & Pública Estadual & Universidade \\
\hline & \begin{tabular}{|l} 
USC \\
\end{tabular} & Privada SFL ${ }^{*}$ & Universidade \\
\hline Bragança Paulista & USF & Privada $\mathrm{SFL}^{*}$ & Universidade \\
\hline Caçapava & FSL & Privada CFL ${ }^{* *}$ & Faculdade \\
\hline \multirow{4}{*}{ Campinas } & PUC & Privada SFL* & Universidade \\
\hline & \begin{tabular}{|l} 
SLMANDIC \\
\end{tabular} & Privada CFL ${ }^{* *}$ & Faculdade \\
\hline & \begin{tabular}{|l} 
UNIP \\
\end{tabular} & Privada $\mathrm{CFL}^{* *}$ & Universidade \\
\hline & \begin{tabular}{|l|} 
UNIAN \\
ANHANGUERA
\end{tabular} & Privada $\mathrm{CFL}^{* *}$ & Universidade \\
\hline Catanduva & IMES-FAFICA & Pública Municipal & Faculdade \\
\hline Descalvado & \begin{tabular}{|l} 
Universidade \\
BRASIL
\end{tabular} & Privada $\mathrm{SFL}^{*}$ & Universidade \\
\hline Fernandópolis & \begin{tabular}{|l} 
Universidade \\
BRASIL
\end{tabular} & Privada $\mathrm{SFL}^{*}$ & Universidade \\
\hline Franca & UNIFRAN & Privada CFL ${ }^{* *}$ & Universidade \\
\hline \multirow{3}{*}{ Guarulhos } & \begin{tabular}{|l|} 
UNIAN \\
ANHANGUERA
\end{tabular} & Privada CFL ${ }^{* *}$ & Universidade \\
\hline & FAG & Privada CFL ${ }^{* *}$ & Faculdade \\
\hline & \begin{tabular}{|l} 
UNG \\
\end{tabular} & Privada SFL ${ }^{*}$ & Universidade \\
\hline Itapeva & FAIT & Privada $\mathrm{CFL}^{* * *}$ & Faculdade \\
\hline Itu & CEUNSP & Privada CFL ${ }^{* *}$ & Centro Universitário \\
\hline Lins & UNIMEP & Privada $\mathrm{SFL}^{*}$ & Universidade \\
\hline Marília & UNIMAR & Privada CFL ${ }^{* *}$ & Universidade \\
\hline \multirow[b]{2}{*}{ Mogi das Cruzes } & UMC & Privada $\mathrm{CFL}^{* * *}$ & Centro Universitário \\
\hline & BRAZ CUBAS & Privada CFL ${ }^{* *}$ & Centro Universitário \\
\hline Ourinhos & UNIFIO & Privada SFL ${ }^{*}$ & Centro Universitário \\
\hline Pindamonhangaba & FUNVIC & Privada $\mathrm{SFL}^{*}$ & Faculdade \\
\hline Piracicaba & UNICAMP & Pública Estadual & Universidade \\
\hline $\begin{array}{l}\text { Presidente } \\
\text { Prudente }\end{array}$ & UNOESTE & Privada $\mathrm{SFL}^{*}$ & Universidade \\
\hline \multirow[b]{2}{*}{ Ribeirão Preto } & USP & Pública Estadual & Universidade \\
\hline & UNAERP & Privada SFL ${ }^{*}$ & Universidade \\
\hline Santa Fé do Sul & FUNEC & Pública Municipal & Centro Universitário \\
\hline \multirow{2}{*}{ Santos } & UNISANTA & Privada SFL* & Universidade \\
\hline & UNIMES & Privada SFL ${ }^{*}$ & Universidade \\
\hline São Carlos & UNICEP & Privada $\mathrm{CFL}^{* *}$ & Centro Universitário \\
\hline \multirow{3}{*}{$\begin{array}{l}\text { São José do Rio } \\
\text { Preto }\end{array}$} & UNIRP & Privada CFL ${ }^{* *}$ & Centro Universitário \\
\hline & UNILAGO & Privada SFL ${ }^{*}$ & Faculdade \\
\hline & UNORP & Privada $\mathrm{SFL}^{*}$ & Centro Universitário \\
\hline \multirow{3}{*}{$\begin{array}{l}\text { São José dos } \\
\text { Campos }\end{array}$} & \begin{tabular}{|l|} 
UNIAN \\
ANHANGUERA
\end{tabular} & Privada $\mathrm{CFL}^{* *}$ & Universidade \\
\hline & \begin{tabular}{|l|} 
UNESP \\
\end{tabular} & Pública Estadual & Universidade \\
\hline & \begin{tabular}{|l} 
UNIVAP \\
\end{tabular} & Privada SFL ${ }^{*}$ & Universidade \\
\hline \multirow{3}{*}{ Sorocaba } & \begin{tabular}{|l|} 
UNIAN \\
ANHANGUERA
\end{tabular} & Privada $\mathrm{CFL}^{* *}$ & Universidade \\
\hline & UNIP & Privada CFL ${ }^{* *}$ & Universidade \\
\hline & \begin{tabular}{|l|} 
UNISO \\
\end{tabular} & Privada SFL* & Universidade \\
\hline Taubaté & UNITAU & Pública Municipal & Universidade \\
\hline
\end{tabular}

A Tabela 2 apresenta os resultados dos Cursos de Odontologia no interior paulista com relação à data de início do curso e o número de vagas.

A busca pelo número de cirurgiões-dentistas registrados no CROSP (Conselho Regional de Odontologia de São Paulo) em atividade nas cidades do interior paulista com cursos de graduação em 
Odontologia resultou em 26.397 registros (Tabela 3). Este valor corresponde a $30 \%$ dos profissionais em atividade, já que o estado de São Paulo conta com um total de 88.264 profissionais registrados no CROSP.

Tabela 2: Distribuição dos Cursos de Odontologia no interior paulista por município, instituição, início do curso e número de vagas

\begin{tabular}{|c|c|c|c|}
\hline Município & Instituição & $\begin{array}{l}\text { Início do } \\
\text { Curso }\end{array}$ & Vagas \\
\hline Adamantina & FAI & 2000 & 80 \\
\hline Araçatuba & UNESP & 1954 & 110 \\
\hline \multirow{2}{*}{ Araraquara } & UNESP & 1923 & 75 \\
\hline & \begin{tabular}{|l|} 
UNIARA \\
\end{tabular} & 2003 & 100 \\
\hline Araras & UNIARARAS FHO & 1986 & 180 \\
\hline Avaré & UNIFSP & 2014 & 80 \\
\hline Barretos & UNIFEB & 1984 & 80 \\
\hline \multirow{2}{*}{ Bauru } & USP & 1948 & 50 \\
\hline & USC & 1991 & 80 \\
\hline Bragança Paulista & USF & 1973 & 120 \\
\hline Caçapava & FSL & 2018 & 120 \\
\hline \multirow{4}{*}{ Campinas } & PUC & 1952 & 90 \\
\hline & \begin{tabular}{|l} 
SLMANDIC \\
\end{tabular} & 2004 & 60 \\
\hline & \begin{tabular}{|l|} 
UNIP \\
\end{tabular} & 1997 & 100 \\
\hline & UNIAN ANHANGUERA & 2017 & 120 \\
\hline Catanduva & IMES-FAFICA & 2000 & 50 \\
\hline Descalvado & Universidade BRASIL & 2013 & 100 \\
\hline Fernandópolis & Universidade BRASIL & 2007 & 128 \\
\hline Franca & UNIFRAN & 1997 & 240 \\
\hline \multirow{3}{*}{ Guarulhos } & UNIAN ANHANGUERA & 2017 & 110 \\
\hline & FAG & 2011 & 50 \\
\hline & UNG & 1992 & 600 \\
\hline Itapeva & FAIT & 2017 & 75 \\
\hline Itu & CEUNSP & 2018 & 105 \\
\hline Lins & UNIMEP & 1954 & 80 \\
\hline Marília & UNIMAR & 1978 & 100 \\
\hline \multirow{2}{*}{ Mogi das Cruzes } & UMC & 1969 & 280 \\
\hline & \begin{tabular}{|l|} 
BRAZ CUBAS \\
\end{tabular} & 1997 & 192 \\
\hline Ourinhos & UNIFIO & 2016 & 50 \\
\hline Pindamonhangaba & FUNVIC & 2003 & 100 \\
\hline Piracicaba & UNICAMP & 1967 & 80 \\
\hline Presidente Prudente & \begin{tabular}{|l} 
UNOESTE \\
\end{tabular} & 1974 & 100 \\
\hline \multirow{2}{*}{ Ribeirão Preto } & USP & 1924 & 80 \\
\hline & UNAERP & 1986 & 100 \\
\hline Santa Fé do Sul & FUNEC & 1999 & 80 \\
\hline \multirow{2}{*}{ Santos } & UNISANTA & 1998 & 80 \\
\hline & \begin{tabular}{|l|} 
UNIMES \\
\end{tabular} & 1975 & 100 \\
\hline $\begin{array}{l}\text { São Carlos } \\
\end{array}$ & UNICEP & 2018 & 90 \\
\hline \multirow{3}{*}{$\begin{array}{l}\text { São José do Rio } \\
\text { Preto }\end{array}$} & UNIRP & 2000 & 200 \\
\hline & \begin{tabular}{|l|} 
UNILAGO \\
\end{tabular} & 2018 & 200 \\
\hline & \begin{tabular}{|l|} 
UNORP \\
\end{tabular} & 2007 & 120 \\
\hline \multirow{3}{*}{ São José dos Campos } & \begin{tabular}{|l|} 
UNIAN ANHANGUERA \\
\end{tabular} & 2017 & 180 \\
\hline & \begin{tabular}{|l|} 
UNESP \\
\end{tabular} & 1960 & 80 \\
\hline & \begin{tabular}{|l|} 
UNIVAP \\
\end{tabular} & 1994 & 60 \\
\hline \multirow{3}{*}{ Sorocaba } & UNIAN ANHANGUERA & 2018 & 150 \\
\hline & UNIP & 1997 & 115 \\
\hline & \begin{tabular}{|l|} 
UNISO \\
\end{tabular} & 2016 & 120 \\
\hline Taubaté & UNITAU & 1979 & 160 \\
\hline Total de Vagas & & & 5800 \\
\hline
\end{tabular}

Quando se relaciona a concentração de pessoas por cirurgião-dentista nos municípios estudados (Tabela 3), todos apresentaram densidade inferior tanto ao índice recomendado pela Organização Mundial de Saúde (OMS) ${ }^{9}$ quanto ao Ministério da Saúde do Brasil ${ }^{10}$, respectivamente, um cirurgião-dentista para cada 1500 ou 3000 pessoas.

Cascaes et al. ${ }^{11}$ chamam a atenção para o caráter particular da relação ideal de habitantes por cirurgiões-dentistas face as demandas de cada população e em cada contexto. A densidade número de habitantes/cirurgião-dentista foi maior em Itapeva, (816) e menor em Santos (205).
Tabela 3: Distribuição dos cirurgiões-dentistas, razão habitante/CD e renda per capita em municípios com Cursos de Odontologia no interior paulista

\begin{tabular}{|c|c|c|c|c|}
\hline Município & Instituição & \begin{tabular}{|c|} 
CD por \\
município
\end{tabular} & $\begin{array}{c}\text { Habitantes } \\
\text { por CD }\end{array}$ & $\begin{array}{c}\text { PIB per } \\
\text { capita } \\
\text { em reais }\end{array}$ \\
\hline Adamantina & FAI & 104 & 325 & 34,013 \\
\hline Araçatuba & UNESP & 731 & 250 & 37,043 \\
\hline \multirow[b]{2}{*}{ Araraquara } & UNESP & \multirow[t]{2}{*}{776} & \multirow[t]{2}{*}{272} & \multirow[t]{2}{*}{40,383} \\
\hline & \begin{tabular}{|l|} 
UNIARA \\
\end{tabular} & & & \\
\hline Araras & UNIARARAS FHO & 317 & 379 & 39,556 \\
\hline Avaré & UNIFSP & 157 & 532 & 29,047 \\
\hline Barretos & UNIFEB & 362 & 311 & 38,127 \\
\hline \multirow{2}{*}{ Bauru } & USP & \multirow[b]{2}{*}{1541} & \multirow{2}{*}{224} & \multirow[b]{2}{*}{36,834} \\
\hline & \begin{tabular}{|l|l} 
USC \\
\end{tabular} & & & \\
\hline Bragança Paulista & USF & 428 & 347 & 32,276 \\
\hline Caçapava & FSL & 134 & 638 & 40,266 \\
\hline \multirow{4}{*}{ Campinas } & PUC & \multirow[t]{4}{*}{3294} & \multirow[t]{4}{*}{331} & \multirow[t]{4}{*}{$\begin{array}{ll}1,219 \\
19\end{array}$} \\
\hline & \begin{tabular}{|l|} 
SLMANDIC \\
\end{tabular} & & & \\
\hline & \begin{tabular}{|l|} 
UNIP \\
\end{tabular} & & & \\
\hline & \begin{tabular}{|l|} 
UNIAN \\
ANHANGUERA
\end{tabular} & & & \\
\hline Catanduva & IMES-FAFICA & 321 & 353 & 33,339 \\
\hline Descalvado & \begin{tabular}{|l} 
Universidade \\
BRASIL
\end{tabular} & 58 & 539 & 43,389 \\
\hline Fernandópolis & \begin{tabular}{|l} 
Universidade \\
BRASIL
\end{tabular} & 204 & 318 & 30,086 \\
\hline Franca & UNIFRAN & 784 & 410 & 29,061 \\
\hline \multirow{3}{*}{ Guarulhos } & $\begin{array}{l}\text { UNIAN } \\
\text { ANHANGUERA }\end{array}$ & \multirow[t]{3}{*}{1971} & \multirow[t]{3}{*}{627} & \multirow[t]{3}{*}{41,497} \\
\hline & \begin{tabular}{|l|} 
FAG \\
\end{tabular} & & & \\
\hline & UNG & & & \\
\hline Itapeva & FAIT & 108 & 816 & 26,778 \\
\hline Itu & CEUNSP & 266 & 586 & 46,661 \\
\hline Lins & UNIMEP & 291 & 247 & 57,130 \\
\hline Marília & UNIMAR & 644 & 339 & 32,537 \\
\hline \multirow[b]{2}{*}{ Mogi das Cruzes } & UMC & \multirow[t]{2}{*}{1179} & \multirow[t]{2}{*}{333} & \multirow[t]{2}{*}{34,753} \\
\hline & BRAZ CUBAS & & & \\
\hline Ourinhos & UNIFIO & 193 & 538 & 25,767 \\
\hline Pindamonhangaba & FUNVIC & 286 & 521 & 42,606 \\
\hline Piracicaba & \begin{tabular}{|l|} 
UNICAMP \\
\end{tabular} & 1073 & 342 & 56,657 \\
\hline $\begin{array}{l}\text { Presidente } \\
\text { Prudente }\end{array}$ & UNOESTE & 640 & 327 & 34,282 \\
\hline \multirow{2}{*}{ Ribeirão Preto } & USP & \multirow[t]{2}{*}{2247} & 273 & 45,789 \\
\hline & \begin{tabular}{|l|} 
UNAERP \\
\end{tabular} & & & \\
\hline Santa Fé do Sul & FUNEC & 124 & 237 & 31,634 \\
\hline & UNISANTA & 2046 & 205 & 51,707 \\
\hline Santos & \begin{tabular}{|l|} 
UNIMES \\
\end{tabular} & & & \\
\hline São Carlos & UNICEP & 562 & 400 & 42,804 \\
\hline & UNIRP & 1285 & 321 & 36,276 \\
\hline $\begin{array}{l}\text { Sáo José do Rio } \\
\text { Preto }\end{array}$ & \begin{tabular}{|l|} 
UNILAGO \\
\end{tabular} & & & \\
\hline & UNORP & & & \\
\hline São José dos & $\begin{array}{l}\text { UNIAN } \\
\text { ANHANGUERA }\end{array}$ & 1918 & 333 & 54,876 \\
\hline Campos & \begin{tabular}{|l|} 
UNESP \\
\end{tabular} & & & \\
\hline & \begin{tabular}{|l|} 
UNIVAP \\
\end{tabular} & & & \\
\hline & $\begin{array}{l}\text { UNIAN } \\
\text { ANHANGUERA }\end{array}$ & 1463 & 407 & 48,519 \\
\hline Sorocaba & \begin{tabular}{|l|} 
UNIP \\
\end{tabular} & & & \\
\hline & \begin{tabular}{|l|} 
UNISO \\
\end{tabular} & & & \\
\hline Taubaté & UNITAU & 890 & 316 & 47,683 \\
\hline Total de CDs & & 26.397 & & \\
\hline
\end{tabular}

O PIB per capita e o número de cirurgiõesdentistas não mostraram correlação positiva. Em municípios com valores próximos de PIB per capita, tais como Ribeirão Preto (R $\$ 45,789)$ e Itu $(\mathrm{R} \$ 46,661)$, os profissionais registrados diferem amplamente, com 2247 cirurgiões-dentistas registrados em Ribeirão Preto contra 266 profissionais em Itu, com um cirurgião-dentista para cada 273 habitantes em Ribeirão Preto e um profissional para cada 586 habitantes em Itu (Tabela 3). Logo, o PIB per capita não reponde isoladamente pela distribuição dos profissionais nos 
municípios do interior paulista com Cursos de Odontologia.

O município do interior paulista com curso de Odontologia e maior número de cirurgiõesdentistas registrados no CROSP é Campinas, seguido por Ribeirão Preto e Santos, com 3294, 2247 e 2046 profissionais, respectivamente (Tabela 3). Quando considerados seus valores de PIB per capita, Campinas ocupa a $5^{\mathrm{a}}$ posição, Ribeirão Preto a posição de número nove e Santos o quarto lugar. Esses municípios são exemplos de polos do interior paulista que se metropolizaram, dentro de uma ótica de tecitura urbana em escala regional ${ }^{12,13}$ (Lencioni, 2003, Marandola Jr). Campinas e Ribeirão Preto foram classificadas em 2017 pelo IBGE $^{14}$ como duas das onze regiões intermediárias do estado de São Paulo, respondendo, por 87 e 64 municípios, respectivamente. Santos, por outro lado, se destaca como uma das duas regiões imediatas da região geográfica intermediária de São Paulo, respondendo por 11 municípios.

Esse processo de desenvolvimento no interior paulista coincide com a modernidade líquida, iniciada a partir dos anos 90 com a gênese de um novo modelo capitalista, repercutindo sobre o modo de vida e escolha das pessoas, valores, identidade, forma de estruturação do espaço urbano regional, relações do mercado de trabalho e da própria cadeia produtiva $^{13,15,16}$.

Segundo a Fundação Getúlio Vargas (FGV), as principais características dessas regiões são capital humano, qualidade de vida, instituições, ambiente de negócios, mercados e recursos naturais, além de educação básica, educação superior e profissionalizante; infraestrutura social; sustentabilidade; saúde; desempenho do setor público; logística (setor viário eficiente), sofisticação dos negócios; inovação; tamanho do mercado; mercado de bens; mercado de trabalho; recursos de energia renovável; agricultura e extrativismo. Lima e Lima $^{18}$ apontam que as regiões com PIB per capita elevado acabam exercendo um efeito cath-up em seu entorno econômico.

Marandola $\mathrm{Jr}$ et al. $^{19}$ destacam que a perspectiva de inserção em uma determinada região (sede ou seu entorno) reverbera repercussões na mobilidade pessoal para acesso aos bens de consumo, à cultura e aos serviços urbanos. Importante considerar que a acessibilidade é um elemento virtual, já que não precisa se efetivar para fazer parte da experiência, mas que interfere na constituição da trajetória pessoal do indivíduo.

Segundo o $\mathrm{IBGE}^{20}$, municípios paulistas que abrigam centros universitários e empresas, principalmente aquelas voltadas para alta tecnologia e inovação, potencializam a capacidade de produzir bens, prestar serviços, estabelecer parcerias e gerir recursos. Nesse sentido, observa-se um movimento de aumento da demanda por profissionais mais especializados.

Silva e Simões ${ }^{21}$ argumentam que uma base estrutural que combina produção de conhecimento científico e tecnológico a partir da interação entre as universidades e centros de pesquisa com atividades industriais é fator decisivo para o avanço econômico de uma região.

\section{CONCLUSÃO}

Os Cursos de Odontologia instalados no interior paulista embora dinamizem a economia dos 32 municípios onde estão instalados, não respondem isoladamente pela concentração de cirurgiõesdentistas nesses espaços geográficos. Outros fatores tais como capital humano, qualidade de vida, instituições, ambiente de negócios, mercados e recursos naturais, além de educação básica, educação superior e profissionalizante; infraestrutura social; sustentabilidade; saúde; desempenho do setor público; logística (setor viário eficiente), sofisticação dos negócios; inovação; tamanho do mercado; mercado de bens; mercado de trabalho; recursos de energia renovável; agricultura e extrativismo.

\section{REFERÊNCIAS}

1. Bleicher L. Autonomia ou assalariamento precário? O trabalho dos cirurgiões-dentistas na cidade de Salvador [tese]. Salvador: Universidade Federal da Bahia UFBA; 2011.

2. Schneider L. Educação e desenvolvimento: um estudo do impacto econômico da universidade federal no município de Santa Maria (RS). Santa Maria: UNIFRA; 2002.

3. Guimarães SK. Transformações científicas e tecnológicas e implicações econômicosociais. Sociologias. 2017;19(46):16-29.

4. Bovo JM, Silva RT, Guzzi VS. A inserção social da UNESP de Araraquara: sua importância na economia do município e na prestação de serviços à comunidade. Perspectivas. 1996;19:71-85.

5. Cardoso AL, Vieira ALS, Machado MH. Mercado de Trabalho dos Odontólogos no Brasil. Divulg Saúde Debate. 2010;45(5):71-9.

6. Conselho Regional de Odontologia de São Paulo (CROSP). Disponível em http://www. crosp. org. br/ intranet/ estatisticas/ estMunicipios.php. Acesso em 02 de abril de 2018.

7. Ministério da Educação e Cultura (e-MEC). Disponível em http://emec.mec.gov.br/. Acesso em 02 de abril de 2018.

8. Portal de Estatísticas do estado de São Paulo (SEADE). Disponível em (https://www.seade. gov.br/produtos/pib-municipal/). Acesso em 02 de abril de 2018.

9. Organização Mundial da Saúde (OMS). WHO Global Health Workforce Statistics. Disponível em https://www.who.int/hrh/statistics/ hwfstats/en/. Acesso em 20 de abril de 2018. 
CONFLITO DE INTERESSES

10.Brasil. Condições de saúde bucal da população brasileira: resultados principais. Brasília: Coordenação Nacional de Saúde Bucal, Departamento de Atenção básica, Secretaria de Atenção à Saúde, Ministério da Saúde. 2010. Disponível em http://dab.saude.gov.br/ CNSB/sbbrasil/arquivos/projeto_sb2010_relatorio final.pdf. Acesso em 20 de abril de 2018.

11. Cascaes AM, Dotto L, Bomfim RA. Tendências da força de trabalho de cirurgiões-dentistas no Brasil, no período de 2007 a 2014: estudo de séries temporais com dados do Cadastro Nacional de Estabelecimentos de Saúde. Epidemiol Serv Saúde. 2018;27(1):e201723615.

12.Lencioni S. Uma nova determinação do urbano: o desenvolvimento do processo de metropolização do espaço. In: Carlos, A. F. A.; Lemos, A. I. (Orgs.). Dilemas urbanos: novas abordagens sobre a cidade. São Paulo: Contexto, 2003.

13. Marandola Jr E, Hogan DJ. Vulnerabilidades e riscos: entre Geografia e Demografia. R bras Est Pop. 2005;22(1):29-53.

14.Instituto Brasileiro de Geografia e Estatística (IBGE). Disponível em ftp://geoftp.ibge.gov.br/ organizacao_do_territorio/estrutura_territorial/divi sao_territorial/. Acesso em 15 de abril de 2018.

15.Bauman Z. Modernidade líquida. Rio de Janeiro: Jorge Zahar; 1991.

16. Senett R. A Cultura do novo capitalismo. Rio de Janeiro: Record; 2006.

17.Fundação Getúlio Vargas (FGV). In: Blumenschein FN. Estudos Econômicos. 2015

18.Lima RCA, Lima JPL. Distribuição do PIB per capita nas microrregiões brasileiras: uma análise exploratória espacial. IPEA ppp. 2016;47:305-29.

19. Marandola Jr E, Guedes GR, Silva RB. Perfis de mobilidade nas regiões metropolitanas do interior de São Paulo. Papeles de Población CIEAP/UAEM. 2010;16(66):210-26.

20.Instituto Brasileiro de Geografia e Estatística (IBGE). Agência de notícias. Disponível em https://agenciadenoticias.ibge.gov.br/. Acesso em 15 de abril de 2018.

21. Silva L, Simões R. Oportunidades tecnológicas e produção científica: uma análise microrregional para o Brasil. Eure (Santiago). 2004; 30(90):85-102.
Os autores declaram não haver conflitos de interesse.

AUTOR PARA CORRESPONDENCIA

Maria Cristina Rosifini Alves Rezende

cristina.rosifini@unesp.br

Submetido em 16/08/2018

Aceito em 12/09/2018 